\section{Cureus}

Received 04/03/2017

Review began 05/13/2017

Review ended 06/06/2017

Published 06/20/2017

\section{(c) Copyright 2017}

Schiedo et al. This is an open access article distributed under the terms of the Creative Commons Attribution License CC-BY 3.0., which permits unrestricted use, distribution, and reproduction in any medium, provided the original author and source are credited.

\title{
Lumbar Spinal Candida Glabrata Treated Without Surgical Intervention: A Case Report
}

Ryan M. Schiedo ${ }^{1}$, William Lavelle ${ }^{2}$, Mike H. Sun ${ }^{3}$

1. Medical Student, Suny Upstate Medical University, Syracuse, NY 2. Department of Orthopedic Surgery, SUNY Upstate Medical University 3. Department of Orthopedic Surgery, SUNY Upstate Medical University, New York, USA

$\square$ Corresponding author: William Lavelle, lavellwf@yahoo.com

Disclosures can be found in Additional Information at the end of the article

\section{Abstract}

Candida glabrata is a low virulent commensal fungal organism that, rarely, can cause osteomyelitis. Diagnosis of such an infection is often difficult as the case typically presents with an insidious onset of back pain and minimally elevated biomarkers of inflammation. Furthermore, it is difficult to eradicate and often resistant to common antifungals.

A 61-year-old man presented with an eight-month history of persistent low back pain which had unsuccessfully been managed by his primary care physician. He had a past surgical history of gastric by-pass complicated by adhesions, ulceration, and perforation with an infection of Candida glabrata that had been treated with intravenous micafungin. Radiological examination showed degenerative changes with suspicion of osteomyelitis and a computerized tomography (CT)-guided biopsy provided tissue samples with subsequent positive cultures for Candida glabrata. The patient was admitted for fungal osteomyelitis with Candida glabrata, treated with intravenous micafungin, and his infection was resolved after six months. At two-year follow-up his back pain has been resolved and no infection was present. In a patient with osteoarticular pain and a previous history of candidal infection with possible candidemia, one should maintain suspicion for fungal osteomyelitis.

Categories: Dermatology, Infectious Disease, Orthopedics

Keywords: candida glabrata, vertebral osteomyelitis, low back pain, micafungin, osteoarticular pain, infection, non-surgical treatment

\section{Introduction}

Candida glabrata is a low virulent commensal fungal organism present on human mucosal tissues [1]. Candida glabrata is a rare opportunistic pathogen known to cause osteomyelitis. Diagnosing Candida glabrata osteomyelitis is challenging, as patients with lumbar osteomyelitis typically present with an insidious onset of back pain and minimally elevated biomarkers of inflammation [2]. It is paramount to accurately identify Candida glabrata from other candida species, as it has an increased natural resistance to antifungal agents, making successful treatment difficult [3].

\section{Case Presentation}

Informed consent was obtained from the patient. We saw a 61-year-old man with an eightmonth history of persistent low back pain that woke him from sleep. He denied numbness, 
tingling, and bowel and bladder incontinence. He also denied any history of fever, chills, cough, vomiting, diarrhea, or night sweats. On a visual analog scale (VAS), he ranked his pain 6/10. His primary care physician managed his chronic pain unsuccessfully with hydrocodoneacetaminophen, a cane, and physical therapy.

His past medical history comprised depression, hypertension, anxiety, and paranoid schizophrenia, but was negative for HIV and immunodeficiency. His surgical history included a Roux-en-Y gastric by-pass performed 10 months ago, complicated by adhesions, ulceration, and perforation. An exploratory laparotomy was performed at that time and an enterocutaneous fistula was discovered. It was infected with Candida glabrata which was later found to be partially resistant to fluconazole. The fistula was repaired and he was treated with intravenous micafungin over the course of several weeks.

On physical examination, his lumbar range of motion with twisting and bending was painful at end range. Palpation of the spinal processes and paraspinal region elicited tenderness. His neurological examination was unremarkable. Lab values: white blood cell count (WBC), 8,700 cells $/ \mathrm{mm}^{3}$ with $68 \%$ neutrophils, $20 \%$ lymphocytes, $8 \%$ monocytes, $3 \%$ eosinophils, and $1 \%$ basophils; hemoglobin ( $\mathrm{Hb}), 12.8 \mathrm{~g} / \mathrm{dL}$; hematocrit, $37.8 \%$; platelets $212,000 / \mathrm{mm}^{3}$; erythrocyte sedimentation rate (ESR), $31 \mathrm{~mm} / \mathrm{hr}$; C-reactive protein (CRP), $13.2 \mathrm{mg} / \mathrm{L}$; and liver function tests were within normal limits.

Plain radiographs revealed degenerative changes without evidence of fracture (Figure 1). Magnetic resonance imaging (MRI) raised the suspicion of osteomyelitis involving L2-3 and L34 with a possible abscess in the L3-4 disk space (Figure 2). A computerized tomography (CT)guided biopsy provided tissue which registered positive cultures for Candida glabrata resistant to fluconazole, itraconazole, and posaconazole, but sensitive to micafungin and amphotericin B. No anaerobes, acid-fast bacilli, or aerobic organisms were present in the bioptic material. 


\section{Cureus}

FIGURE 1: Lateral X-ray of the lumbar spine. There is evidence of erosive change on the anterior and superior plate of L4 that was not previously present. There is loss of disc height at L3L4 and grade 1 anterolisthesis of L4-L3, findings suggestive of osteomyelitis and discitis. 


\section{Cureus}

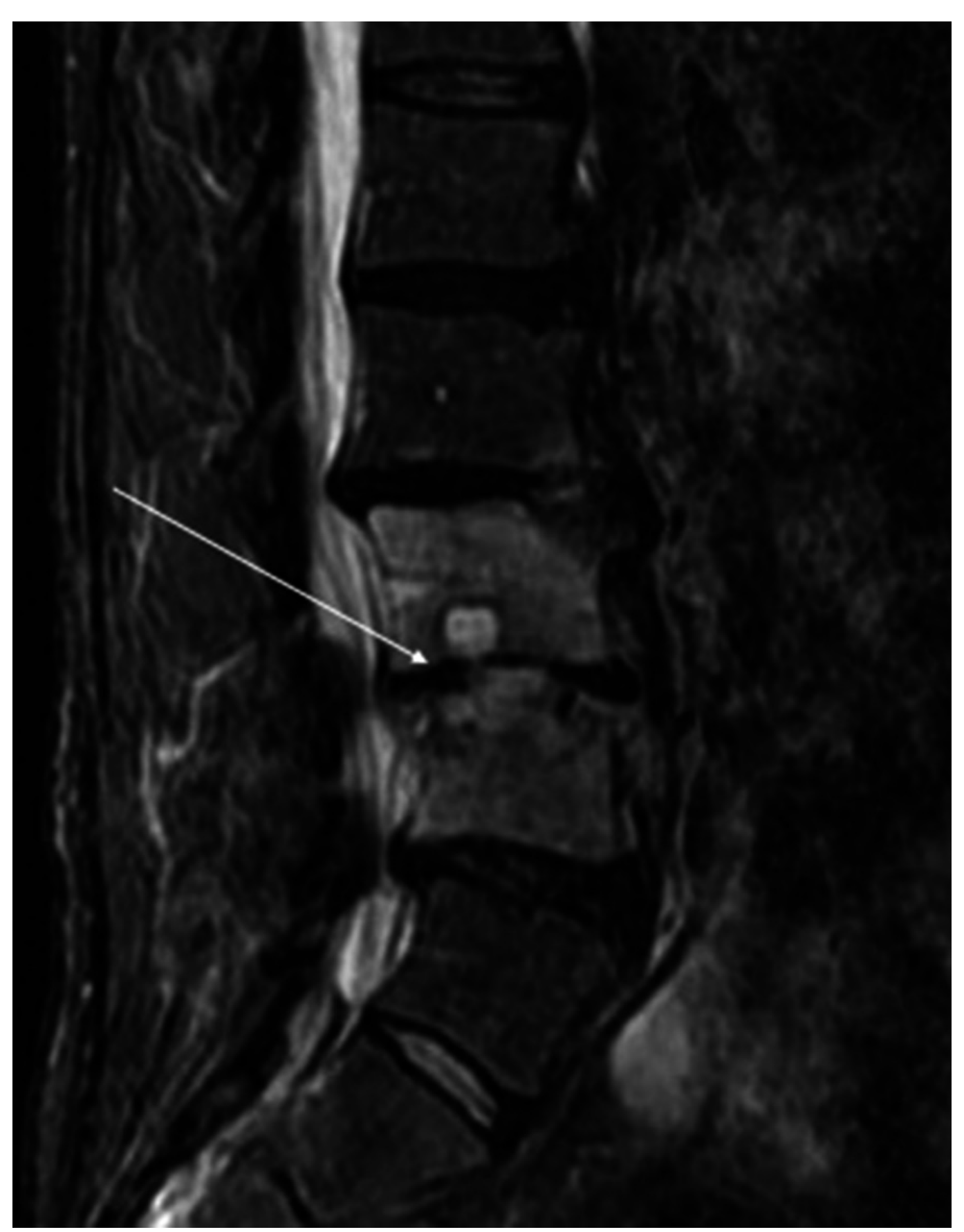

FIGURE 2: Non-contrast T2 MRI of the spine. There is evidence of osteomyelitis at the L2-L3 and L3-L4 intervertebral levels with abscess in the disc space at the L3-L4 and anterior aspect of the disc at L2-L3.

The patient was admitted for fungal osteomyelitis with Candida glabrata and treatment with intravenous micafungin was begun. After six months of treatment, his infection resolved and he was discharged. At two-year follow-up, his back pain has fully resolved (VAS Analog Scale 1/10) and an MRI revealed resolution of the infection (Figure 3). 


\section{Cureus}

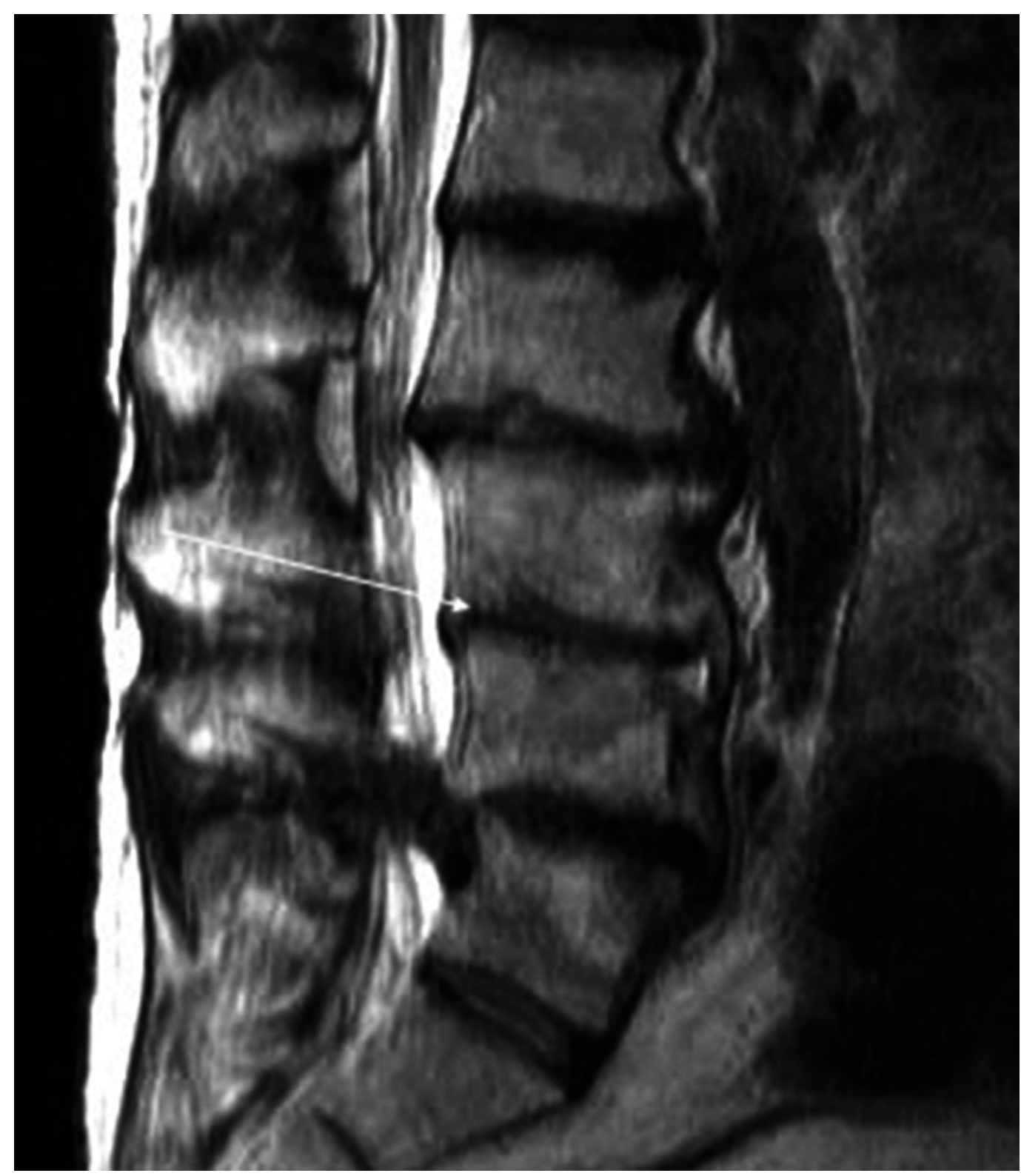

FIGURE 3: MRI without contrast. This image was taken nearly a year after he was discharged from the hospital. The anterior edema in the endplate is no longer present on the T2 MRI scan. The patient is asymptomatic with no signs of infection.

\section{Discussion}

Osteomyelitis with Candida glabrata is a rare infection that is difficult to treat. The most commonly identified portal of entry for Candida glabrata fungemia is through the abdomen [4], a finding consistent with our case. His initial abdominal infection grew Candida glabrata partially resistant to fluconazole. Ten months later, a biopsy of his lumbar spine showed that the organism was fully resistant to fluconazole, itraconazole and posaconazole. It is likely that the vertebrae were seeded at the time of the initial Candida glabrata infection. 
reported in patients with candidal vertebral osteomyelitis. In a study of 207 patients with fungal osteomyelitis, 87\% of patients had elevated ESR [5]. Our patient had a moderately elevated ESR and CRP, but lacked an elevated WBC or fever. The lack of sepsis warranted a search for a less common pathogen. In our case, the first biopsy grew Candida glabrata; however, after reviewing the literature it was found this is not always the case. Miller and Mejicano suggested that if the first biopsy is negative, but there is a high suspicion for osteomyelitis, then a second biopsy should be performed; and if that is also negative, surgical exploration should be undertaken [5]. Identifying the organism is paramount, as therapy for mycobacterial and fungal osteomyelitis are vastly different.

The primary pathophysiological mechanism of vertebral osteomyelitis in adults is hematogenous dissemination [6]. When infection is spread hematogenously, the most frequent site of bony involvement is the lumbar spine [7]. Patients typically have an effusion and tenderness with joint range of motion [8]. All of these findings were consistent with our case.

\section{Conclusions}

Diagnosing candida osteomyelitis is difficult as it is an uncommon pathological organism. When a patient has osteoarticular pain and a previous history of candidemia, one should maintain suspicion for a subsequent osteomyelitis. This holds true even if the initial infection was months or years ago. Culture and antibiogram sensitivities should be performed to ensure proper treatment of the infection.

\section{Additional Information \\ Disclosures}

Human subjects: Consent was obtained by all participants in this study. Conflicts of interest: In compliance with the ICMJE uniform disclosure form, all authors declare the following: Payment/services info: All authors have declared that no financial support was received from any organization for the submitted work. Financial relationships: William Lavelle declare(s) a grant from DePuy Spine. William Lavelle declare(s) a grant from Medtronic. William Lavelle declare(s) a grant from IntegraLife. William Lavelle declare(s) a grant from Sigmus, Inc. William Lavelle declare(s) a grant from K2M, Inc. William Lavelle declare(s) a grant from Spinal Kinetics, Inc. William Lavelle declare(s) a grant from Providence Technologies. William Lavelle declare(s) a grant from Stryker Spine. William Lavelle declare(s) a grant from Vertebral Technologies, Inc. Other relationships: All authors have declared that there are no other relationships or activities that could appear to have influenced the submitted work.

\section{References}

1. Haley LD: Yeasts of medical importance. Am J Clin Pathol. 1961, 36:227-34. 10.1093/ajcp/36.3.227

2. Gamaletsou MN, Kontoyiannis DP, Sipsas NV, et al.: Candida osteomyelitis: analysis of 207 pediatric and adult cases (1970-2011). Clin Infect Dis. 2012, 55:1338-51. 10.1093/cid/cis660

3. Komshian SV, Uwaydah AK, Sobel JD, et al.: Fungemia caused by candida species and torulopsis glabrata in the hospitalized patient: frequency, characteristics, and evaluation of factors influencing outcome. Rev Infect Dis. 1989, 11:379-90. 10.1093/clinids/11.3.379

4. GumboT, Isada CM, Hall G, et al. : Candida glabrata fungemia: clinical features of 139 patients . Medicine (Baltimore). 1999, 78:220-7.

5. Miller DJ, Mejicano GC: Vertebral osteomyelitis due to candida species: case report and literature review. Clin Infect Dis. 2001, 33:523-30. 10.1086/322634

6. Waldvogel FA, Medoff G, Swartz MN: Osteomyelitis: a review of clinical features, therapeutic considerations and unusual aspects(second of three parts). N Engl J Med. 1970, 282:260-6. 10.1056/NEJM197001292820507

7. Kohli R, Hadley S: Fungal arthritis and osteomyelitis. Infect Dis Clin North Am. 2005, 19:831- 


\section{Cureus}

51. 10.1016/j.idc.2005.08.004

8. Bariteau JT, Waryasz GE, McDonnell M, et al.: Fungal osteomyelitis and septic arthritis. J Am Acad Orthop Surg . 2014, 22:390-401. 10.5435/JAAOS-22-06-390 